\title{
BRCA1 ubiquitinates its phosphorylation-dependent binding partner CtIP
}

\author{
Xiaochun Yu, ${ }^{1,3}$ Shuang Fu, ${ }^{2,3}$ Maoyi Lai, ${ }^{1}$ \\ Richard Baer, ${ }^{2,5}$ and Junjie Chen ${ }^{1,4}$ \\ ${ }^{1}$ Department of Oncology, Mayo Clinic and Foundation, \\ Rochester, Minnesota 55905, USA; ${ }^{2}$ Institute for Cancer \\ Genetics, Department of Pathology, Columbia University, \\ New York, New York 10032, USA
}

BRCA1 (Breast Cancer Susceptibility Gene 1) possesses an N-terminal Ring domain and tandem C-terminal BRCT motifs. While the Ring domain has E3 ubiquitin ligase activity, the BRCA1 BRCT domains specifically recognize phospho-serine motifs. Here, we demonstrate that BRCA1 Ring domain catalyzes CtIP ubiquitination in a manner that depends on a phosphorylation-mediated interaction between CtIP and BRCA1 BRCT domains. The BRCA1-dependent ubiquitination of CtIP does not target CtIP for degradation. Instead, ubiquitinated CtIP associates with chromatin following DNA damage and participates in G2/M checkpoint control. Thus, we propose that BRCA1 can regulate the functions of its substrates through nonproteasomal pathways that do not involve substrate degradation.

Supplemental material is available at http://www.genesdev.org.

Received March 20, 2006; revised version accepted May 2, 2006.

\section{Results and Discussion}

The BRCA1 gene (Breast Cancer Susceptibility Gene 1) encodes a polypeptide of 1863 amino acids that contains an N-terminal Ring domain and tandem C-terminal BRCT domains. The Ring domain of BRCA1 has E3 ubiquitin ligase activity (Lorick et al. 1999; Brzovic et al. 2001; Hashizume et al. 2001; Ruffner et al. 2001; Baer and Ludwig 2002; Chen et al. 2002; Xia et al. 2003; Sato et al. 2004; Starita et al. 2004, 2005; Kleiman et al. 2005), while the BRCT domain has recently been demonstrated to be a phospho-protein interaction domain (Manke et al. 2003; Rodriguez et al. 2003; Yu et al. 2003; Glover et al. 2004). Breast cancer-associated mutations have been found in both the Ring and BRCT domains of BRCA1, suggesting that these two regions are critical for BRCA1 tumor suppression. To explore whether these domains may function together in a coordinated fashion, we examined whether a BRCA1 BRCT domain-binding protein CtIP would be ubiquitinated in a BRCA1-dependent manner.

[Keywords: BRCA1; CtIP; ubiquitination; DNA damage; phosphorylation; BRCT domain]

${ }^{3}$ These authors contributed equally to this work.

Corresponding authors.

${ }^{4}$ E-MAIL chen.junjie@mayo.edu; FAX (507) 284-3906.

${ }^{5}$ E-MAIL rb670@columbia.edu; FAX (212) 851-5267.

Article is online at http://www.genesdev.org/cgi/doi/10.1101/gad.1431006.
CtIP is a phosphorylation-dependent binding partner of the BRCA1 BRCT domain (Wong et al. 1998; Yu et al. 1998; Yu and Chen 2004; Varma et al. 2005). It transiently interacts with BRCA1 in G2 phase, and participates in BRCA1-dependent G2/M checkpoint control (Yu and Chen 2004; Greenberg et al. 2006). To determine whether CtIP is ubiquitinated in vivo, we transfected $293 \mathrm{~T}$ cells with vectors encoding HA-tagged ubiquitin. As shown in Figure 1A, CtIP was clearly conjugated with polyubiquitin chains, suggesting that CtIP is ubiquitinated in vivo. Next, we explored whether BRCA1 participates in CtIP ubiquitination. To do so, we used BRCA1-deficient HCC1937 cells and HCC1937 cells reconstituted with wild-type BRCA1 (HCC1937-BRCA1 cells) (Yu et al. 2003). While endogenous CtIP was readily ubiquitinated in HCC1937-BRCA1 cells, CtIP ubiquitination was barely detectable in HCC1937 cells (Fig. 1B), suggesting that wild-type BRCA1 is required for the ubiquitination of endogenous CtIP.

To demonstrate that BRCA1 directly ubiquitinates CtIP, we also performed in vitro ubiquitination assays. Full-length CtIP, BRCA1, and BARD1 were generated and purified from sf 9 cells infected with corresponding baculoviruses. In the presence of E1 ubiquitin activating enzyme and E2 ubiquitin conjugating enzyme (UbcH5c), CtIP was ubiquitinated by wild-type BRCA1 (Fig. 1C; see also Supplementary Fig. S1). And as expected, wild-type BRCA1 is also autoubiquitinated in vitro (Fig. 1C). We mutated Ile26 to Ala (I26A) in the BRCA1 Ring domain. This I26A mutant does not abolish the tertiary structure of the BRCA1 Ring domain but specifically disrupts the contact site of the BRCA1 Ring domain with E2 ubiquitin conjugase (Brzovic et al. 2003). Therefore, the I26A mutant of BRCA1 still interacts with its Ring domainbinding partner BARD1 but specifically loses its E3 ligase activity. As shown in Figure 1C, the I26A mutant does not ubiquitinate CtIP in vitro, suggesting that the ubiquitination of CtIP observed in these reactions is specifically dependent on the E3 ligase activity of BRCA1.

Polyubiquitination often serves as a signal for protein degradation. To characterize the potential biological consequences of CtIP ubiquitination, we compared the stability of CtIP in HCC1937 cells and HCC1937BRCA1 cells. Interestingly, when we blocked de novo CtIP synthesis in these cells with cycloheximide, the rate of CtIP degradation remained constant regardless of BRCA1 status, suggesting that the BRCA1-dependent ubiquitination of CtIP is not associated with CtIP degradation (Supplementary Fig. S2). Both HCC1937 cells and HCC1937-BRCA1 cells were also treated with MG132, a proteasome inhibitor. As a control, it blocked ubiquitination-mediated Aurora B degradation (Supplementary Fig. S2; Nguyen et al. 2005). However, the halflife of CtIP still remained unchanged, suggesting that CtIP degradation is independent of the proteasome pathway (Supplementary Fig. S2).

Much evidence suggests that BRCA1 participates in various aspects of the DNA damage response in a manner that requires both the Ring and BRCT domains of BRCA1 (Scully et al. 1999; Ruffner et al. 2001; Morris and Solomon 2004). We have also shown that CtIP participates in BRCA1-dependent G2/M checkpoint control following DNA damage (Yu and Chen 2004). Therefore, we investigated whether CtIP ubiquitination is regulated 
A
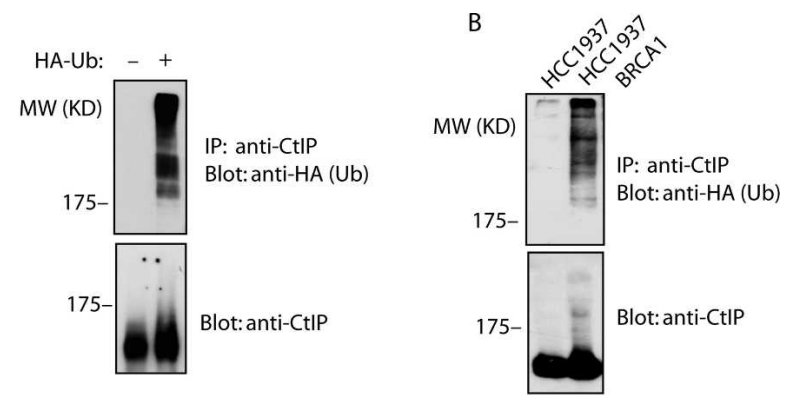

C

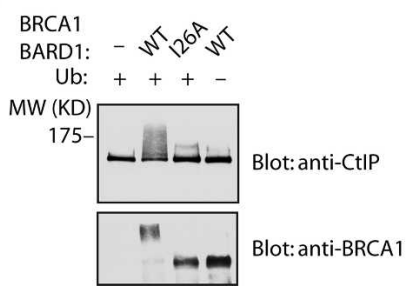

Figure 1. BRCA1 ubiquitinates phosphorylated CtIP in vivo and in vitro. (A) CtIP is ubiquitinated in vivo. $293 \mathrm{~T}$ cells were transfected with or without plasmid encoding HA-tagged ubiquitin (Ub). (Top panel) Ubiquitination of CtIP was analyzed by immunoprecipitation and immunoblotting with indicated antibodies. (Bottom panel) Cell lysates were also blotted with anti-CtIP antibodies. $(B)$ The intact BRCA1 BRCT domain is required for CtIP ubiquitination. HCC1937 cells or HCC1937-BRCA1 cells were transfected with HA-Ub. Endogenous CtIP was significantly ubiquitinated in HCC1937-BRCA1 cells, but not in HCC1937 cells. (C) CtIP is ubiquitinated by BRCA1 in vitro. Full-length CtIP was incubated with wild-type BRCA1 or enzymatically inactive I26A mutant. The reaction products were then fractioned by SDS-PAGE and detected by immunoblotting with indicated antibodies.

by DNA damage and how it participates in the DNA damage response. Both HCC1937 and HCC1937-BRCA1 cells were treated with $\gamma$-irradiation. Although, we didn't observe any change of CtIP ubiquitination in the soluble faction of $0.5 \%$ NP40 lyses buffer, interestingly ubiquitinated CtIP was detected in the chromatin fraction only following DNA damage (Fig. 2A). Moreover, this effect was observed in HCC1937-BRCA1 cells but not in HCC1937 cells, suggesting that BRCA1 is required for chromatin association and ubiquitination of endogenous CtIP following DNA damage. As expected, a significant portion of BRCA1 associated with chromatin fractions following DNA damage. Nearly $50 \%$ of CtIP were ubiquitinated and associated with chromatin following DNA damage (Fig. 2B; see also Supplementary Fig. S3). To exclude the possibility of antibody crossreactions, we repeated these experiments using 293T cells stably expressing Flag-tagged CtIP. Again, we found that a fraction of ubiquitinated CtIP was tightly associated with chromatin following $\gamma$-irradiation (Fig. 2C).

HCC1937 cells only express a truncated form of BRCA1 in which the C-terminal BRCT domain is lost (Tomlinson et al. 1998; Yu et al. 2003). Because of this C-terminal deletion, the truncated BRCA1 of HCC1937 cells cannot interact with CtIP through the BRCT domains (Yu et al. 1998; data not shown). However, the truncated BRCA1 species contains an intact $\mathrm{N}$-terminal Ring domain and should retain its E3 ligase activity. The fact that this species cannot effectively promote CtIP ubiquitination in HCC1937 cells (Fig. 1B) suggests that the BRCA1/CtIP interaction is critical for CtIP ubiqui- tination. Our early studies have shown that the BRCA1 BRCT domain specifically binds the Ser327-phosphorylated forms of CtIP (Yu and Chen 2004). To examine the importance of this phospho-dependent BRCA1/CtIP interaction for CtIP ubiquitination, we used a Ser327-toAla (S327A) mutant of CtIP that fails to associate with BRCA1 (Yu and Chen 2004). Indeed, only the wild type, but not the S327A-mutant, CtIP was ubiquitinated in the chromatin fraction (Fig. 2D), suggesting that a direct interaction between BRCA1 and CtIP is important for the ubiquitination and chromatin association of CtIP in vivo. To confirm that BRCA1 is the E3 ubiquitin ligase that catalyzes CtIP ubiquitination in the chromatin fractions, HCC1937 cells were reconstituted with either wild-type BRCA1 or I26A-mutant BRCA1. Significantly, only wild-type BRCA1, but not the I26A mutant, could promote CtIP ubiquitination in these cells (Fig. 2E), again suggesting that the intact E3 ligase activity of BRCA1 is necessary for the ubiquitination and chromatin association of CtIP.

Since protein ubiquitination may influence its localization, we next examined the subnuclear localization of CtIP before and after DNA damage. The peak of CtIP expression is in G2 phase (Yu and Chen 2004). Therefore, only $\sim 30 \%$ cells showed clearly CtIP staining. CtIP is evenly distributed throughout the nucleus in both untreated HCC1937 cells and untreated HCC1937-BRCA1 cells. However, following $\gamma$-radiation, CtIP relocated to nuclear foci in HCC1937-BRCA1 cells, but not in HCC1937 cells (Fig. 3A). The nuclear foci of CtIP colocalized with $\gamma \mathrm{H} 2 \mathrm{AX}$, a marker of DNA damage sites, and BRCA1 (Fig. 3B; see also Supplementary Fig. S4), suggesting that CtIP relocalizes to the sites of DNA damage. Moreover, only wild-type BRCA1, but not the I26A E3 ligase mutant of BRCA1, restored DNA damage-induced CtIP focus formation in HCC1937 cells (Fig. 3C; see also Supplementary Fig. S5), suggesting that formation of CtIP foci requires the E3 ligase activity of BRCA1. Again, only wild-type CtIP, but not the S327A mutant, translocated to DNA damage sites, suggesting that the BRCA1/ CtIP interaction is also essential for CtIP focus formation (Supplementary Fig. S6). It is likely that the CtIP foci reflect the concentrated localization of chromatinassociated ubiquitinated CtIP following DNA damage, as we observed in Figure 2.

We have previously demonstrated that BRCA1, CtIP, and the BRCA1/CtIP interaction are each required for the transient G2/M checkpoint control (Yu and Chen 2004). Here, we further examined this DNA damage-induced G2/M checkpoint control in HCC1937 cells expressing either wild-type BRCA1 or the I26A mutant. Only cells reconstituted with wild-type BRCA1, but not the I26A mutant, restored G2/M checkpoint control following DNA damage (Fig. 3D), suggesting that BRCA1 E3 ligase activity is also essential for this G2/M checkpoint control. Given the critical role of CtIP in this checkpoint control, CtIP is likely the major substrate of BRCA1 that governs this G2/M checkpoint following DNA damage.

In conclusion, our data show that CtIP is a physiological substrate of the BRCA1 E3 ligase. BRCA1 recruits CtIP through its C-terminal BRCT domains and promotes CtIP ubiquitination through its N-terminal Ring domain. The ubiquitinated CtIP is not targeted for degradation. Instead, ubiquitinated CtIP binds to chromatin following DNA damage and is likely to be involved in 

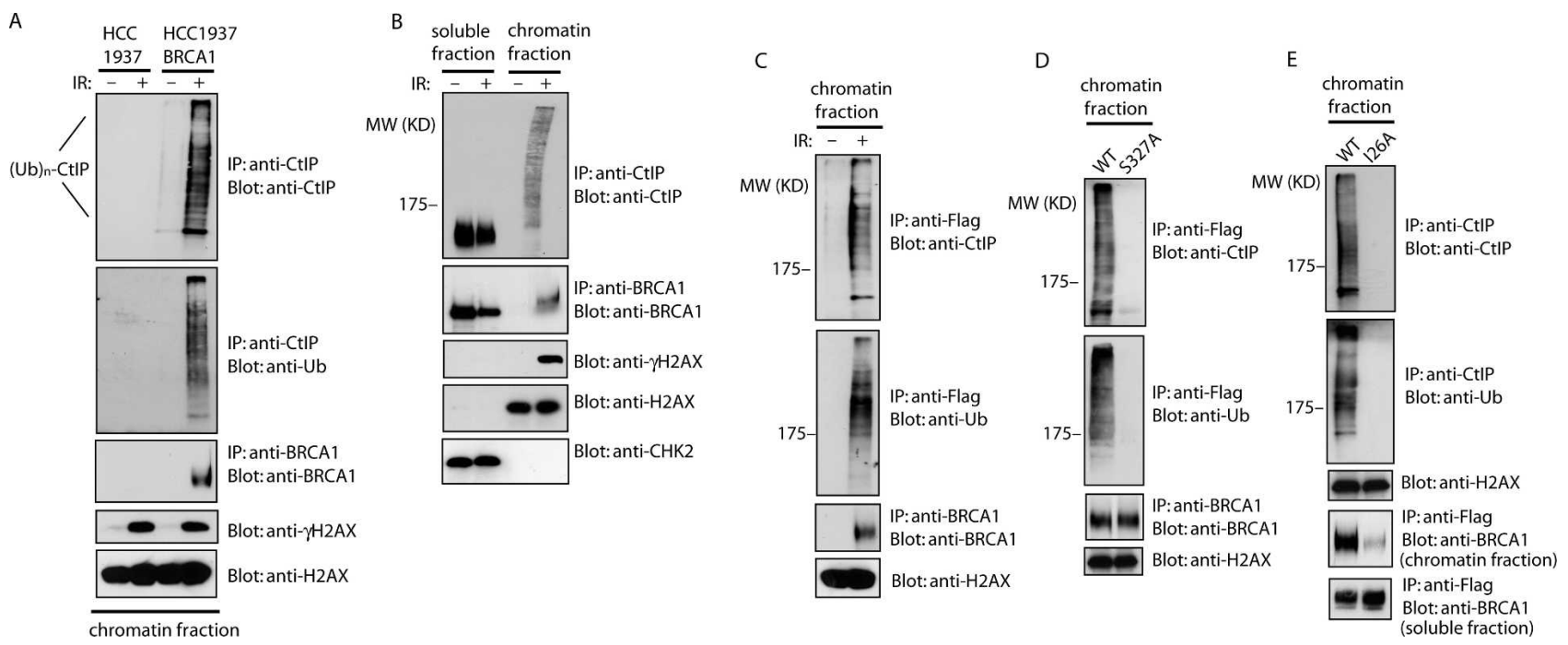

Figure 2. Ubiquitinated CtIP associates with chromatin following DNA damage. HCC1937 cells and HCC1937-BRCA1 cells (A), HCC1937BRCA1 cells $(B)$, or $293 \mathrm{~T}$ cells $(C)$ stably expressing Flag-CtIP were treated with $\gamma$-radiation (10 Gy). Chromatin extraction was prepared as described in Materials and Methods. The lysates were analyzed by immunoprecipitation and immunoblotting with indicated antibodies. $(D)$ Phosphorylation of CtIP S327 site is important for CtIP ubiquitination in chromatin fractions. 293T cells were transfected with vectors encoding wild-type CtIP or S327A mutant and then treated with $\gamma$-radiation (10 Gy). Chromatin extraction was prepared and analyzed. (E) BRCA1 E3 ligase activity is required for CtIP ubiquitination following DNA damage. HCC1937 cells were transfected with wild-type BRCA1 or I26A mutant, and treated with $\gamma$-radiation $(10 \mathrm{~Gy})$. Chromatin extraction was prepared and analyzed.

DNA damage checkpoint control. Thus, we propose that the BRCA1 Ring domain and BRCT domains act together and regulate various BRCA1-dependent functions through protein ubiquitination. This mode of action resembles that of SCF (Skp1-Cullin-F-box) complexes, in which Ring domain subunits ubiquitinate substrates that are recognized in a phospho-dependent manner by F-box-containing proteins.

Our results suggest that CtIP ubiquitination is required for its chromatin binding and damage-induced foci formation, and may play a pivotal role in $\mathrm{G} 2 / \mathrm{M}$ checkpoint control. Interestingly, mono-ubiquitination of FANCD2, a Fanconi Anemia protein, is also essential for its chromatin binding, damage-induced foci formation, S-phase checkpoint activation, and DNA repair (Garcia-Higuera et al. 2001; Taniguchi et al. 2002; Wang et al. 2004; Montes de Oca et al. 2005). Taken together, these data suggest that DNA damage-induced protein mono- and polyubiquitination may serve as another type of signaling cascade that regulates cellular DNA damage responses. Exactly how ubiquitination of FANCD2 or CtIP influences cell cycle checkpoint and DNA repair remains to be determined.

We also attempted to map the BRCA1-dependent ubiquitination sites on CtIP, which contains total 84 Lys residues. However, based on our in vitro ubiquitination assays (data not shown), it appears that CtIP can be ubiquitinated by BRCA1 interchangeably at multiple lysine residues. We hope that the additional ongoing experiments will eventually reveal CtIP ubiquitination sites and further confirm the role of the BRCA1-dependent CtIP ubiquitination in DNA damage checkpoint control.

Beside BRCA1, CtIP has also been shown to interact with another E3 ubiquitin ligase SIAH-1 (Germani et al. 2003). However, whether SIAH-1 really regulates CtIP ubiquitination or degradation has not been demonstrated either in vitro or in vivo (Germani et al. 2003). Thus, the functional significance of CtIP/SIAH-1 interaction is not clear and needs to be further investigated. We have shown previously that the BRCA1 BRCT domain also recognizes phospho-BACH1. However, we did not observe $\mathrm{BACH} 1$ ubiquitination in our experimental systems. One possibility is that there are other partners in the BRCA1/BACH1 complex, and BRCA1 ubiquitinates BACH1-associated proteins instead of directly targeting BACH1 for ubiquitination. Further studies will be needed to test this possibility.

\section{Materials and methods}

Cell culture, antibodies, and plasmids

All cell lines were maintained in RPMI 1640 medium with $10 \%$ fetal calf serum at $37^{\circ} \mathrm{C}$ in $5 \% \mathrm{CO}_{2}(\mathrm{v} / \mathrm{v})$. To measure CtIP degradation, cells were treated with cycloheximide $(40 \mu \mathrm{g} / \mathrm{mL})$ and MG132 $(30 \mu \mathrm{M})$.

Mouse anti-Flag (M2) antibody was purchased from Sigma. Mouse antiHA antibody was purchased from Covance. Polyclonal anti-CtIP antibodies were used for immunoprecipitation and Western blot as described previously (Yu and Chen 2004). Monoclonal mouse anti-CtIP antibody (14-1) was used for immunostaining (Yu and Baer 2000). Rabbit antiphospho-Histone H3 antibodies were purchased from Upstate Biotechnology, Inc.

Wild-type CtIP and S327A mutant were cloned into pCIN4 as described previously (Yu and Baer 2000; Yu and Chen 2004). Wild-type BRCA1 and I26A mutant were cloned into pIRES2-EGFP with one $S$ tag and tandem Flag tag at the $\mathrm{N}$ terminus.

Chromatin extraction preparation

The preparation of chromatin fraction was described previously (Ward and Chen 2001). Briefly, cells were lysed with NETN buffer (0.5\% NP-40, $2 \mathrm{mM}$ EDTA, $50 \mathrm{mM}$ Tris- $\mathrm{HCl}$ at $\mathrm{pH} 8.0$ and $100 \mathrm{mM} \mathrm{NaCl}$ ). The insoluble chromatin fractions were treated with $0.2 \mathrm{M} \mathrm{HCl}$. The soluble extraction was neutralized with $1 \mathrm{M}$ Tris- $\mathrm{HCl}(\mathrm{pH} 8.5)$ and then used for further analysis.

Cell transfection, immunoprecipitation, immunoblotting, and immunostaining

Cell transfection, immunoprecipitation, and immunoblotting were performed with standard protocol as described previously (Yu and Chen 
Yu et al.

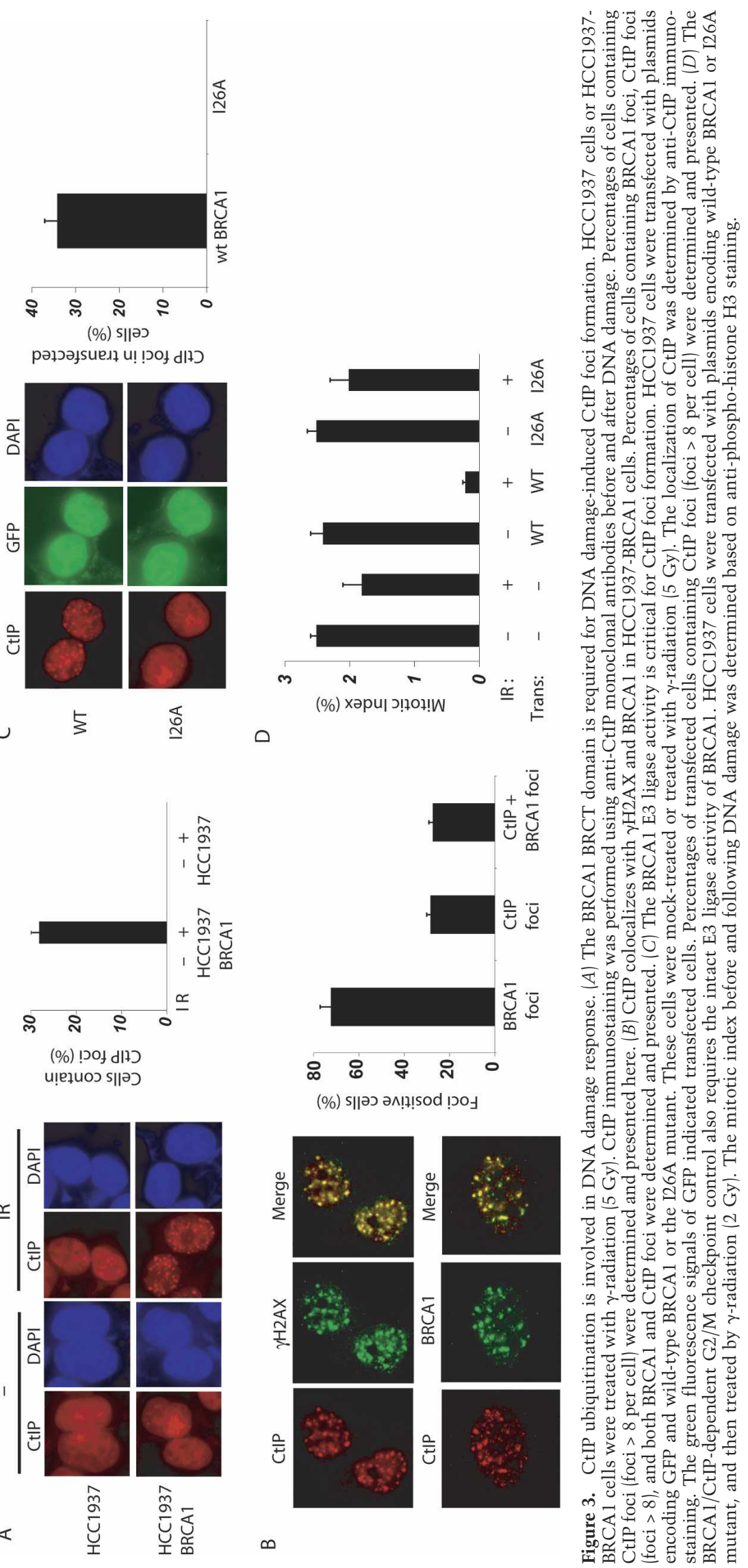


2004). For transfection of HCC1937 cells and HCC1937-BRCA1 cells, we used the electroporation method according to a protocol from the manufacturer (Bio-Rad). In Figure 1, A and B, cells were lysed in NETN buffer with the additional $1 \%$ SDS. Cell lysates were boiled for $10 \mathrm{~min}$, and 10 vol NETN buffer were added to dilute SDS to $0.1 \%$ for further immunoprecipitation.

For immunostaining, cells were treated with 5 Gy of $\gamma$-irradiation. After $2 \mathrm{~h}$, cells were fixed with $3 \%$ paraformaldehyde for $20 \mathrm{~min}$, followed by treatment with $0.5 \%$ Triton X-100 for 5 min. Cells were incubated with anti-CtIP $\mathrm{mAb}$ (14-1) at 1:20 dilution, with anti-BRCA1 antibodies (from Upstate Biotechnology) at 1:500 dilution, with anti-Flag $\mathrm{mAb}$ (M2 from Sigma) at 1:2000 dilution, or with anti- $\gamma \mathrm{H} 2 \mathrm{AX}$ antibodies (from Upstate Biotechnology) at 1:500 dilution and were then incubated with goat anti-mouse IgG- Rhodamine (from Jackson Laboratory) at 1:600 dilution and goat anti-rabbit IgG-FITC (from Jackson Laboratory) at 1:600 dilution. For any quantitative analysis, 1000 cells or transfected cells were counted. Cells with more than eight foci were considered as focipositive cells. Every experiment was repeated at least three times.

\section{G2/M checkpoint assay}

Cells were exposed to $2 \mathrm{~Gy} \gamma$-irradiation and incubated at $37^{\circ} \mathrm{C}$ in $5 \%$ $\mathrm{CO}_{2}(\mathrm{v} / \mathrm{v})$ for one hour. Cells were fixed with $3 \%$ paraformaldehyde and stained with rabbit anti-phospho-Histone $\mathrm{H} 3$ antibody, followed by incubation with Rhodamine-conjugated goat anti-rabbit IgG secondary antibody. Positive-stained cells were observed by immunofluorescence microscopy.

\section{Baculoviral expression}

Baculoviral expression and purification of full-length BRCA1/BARD1 heterodimers were described previously (Wu-Baer et al. 2003). Plasmid for baculoviral expression of the CtIP polypeptides was constructed by inserting the appropriate cDNA sequences into the pVL1393 (Pharmingen). The Fl3-GST-CtIP-6H/pVL1393 plasmid encodes full-length human CtIP with a N-terminal tag of 246 amino acids containing three tandem Flag epitopes and the glutathione $S$-transferase (GST) sequence and a C-terminal tag composed of hexahistidine. To generate recombinant baculoviruses, Sf9 cells were transfected with the appropriate expression plasmid using the BaculoGold transfection kit (Pharmingen). Sf9 spinner cells were then infected with Fl3-GST-CtIP-6H recombinant baculoviruses at a multiplicity of infection of approximately two plaque-forming units per cell. The Fl3-GST-CtIP-6H polypeptides were purified from Sf9 cell lysates using a two-step procedure involving sequential affinity chromatography on a Ni-NTA resin and glutathione-agarose bead (Wu-Baer et al. 2003).

In vitro ubiquitination assay

Twenty-five-nanogram aliquots of purified Fl3-GST-CtIP-6H polypeptides were added to $30 \mu \mathrm{L}$ in vitro ubiquitination reactions (Wu-Baer et al. 2003), and the reaction products were fractionated by SDS-PAGE.

\section{Acknowledgments}

We thank Katherine Minter-Dykhouse for proofreading of this manuscript. This work is supported in part by grants from the National Institute of Health (CA89239 to J.C. and CA97403 to R.B.), Susan G. Komen Breast Cancer Research Foundation, and Eagles Cancer Research Foundation. J.C. is a recipient of the DOD breast cancer career development award (DAMD17-02-1-0472). X.Y. is supported by AACR Cancer Research and Prevention Foundation Fellowship.

\section{References}

Baer, R. and Ludwig, T. 2002. The BRCA1/BARD1 heterodimer, a tumor suppressor complex with ubiquitin E3 ligase activity. Curr. Opin. Genet. Dev. 12: 86-91.

Brzovic, P.S., Rajagopal, P., Hoyt, D.W., King, M.C., and Klevit, R.E. 2001. Structure of a BRCA1-BARD1 heterodimeric RING-RING complex. Nat. Struct. Biol. 8: 833-837.

Brzovic, P.S., Keeffe, J.R., Nishikawa, H., Miyamoto, K., Fox III, D., Fukuda, M., Ohta, T., and Klevit, R. 2003. Binding and recognition in the assembly of an active BRCA1/BARD1 ubiquitin-ligase complex. Proc. Nat1. Acad. Sci. 100: 5646-5651.
Chen, A., Kleiman, F.E., Manley, J.L., Ouchi, T., and Pan, Z.Q. 2002. Autoubiquitination of the BRCA1-BARD1 RING ubiquitin ligase. $J$. Biol. Chem. 277: 22085-22092.

Garcia-Higuera, I., Taniguchi, T., Ganesan, S., Meyn, M.S., Timmers, C., Hejna, J., Grompe, M., and D'Andrea, A.D. 2001. Interaction of the Fanconi anemia proteins and BRCAl in a common pathway. Mol. Cell 7: 249-262.

Germani, A., Prabel, A., Mourah, S., Podgorniak, M.P., Di Carlo, A., Ehrlich, R., Gisselbrecht, S., Varin-Blank, N., Calvo, F., and BruzzoniGiovanelli, H. 2003. SIAH-1 interacts with CtIP and promotes its degradation by the proteasome pathway. Oncogene 22: 8845-8851.

Glover, J.N., Williams, R.S., and Lee, M.S. 2004. Interactions between BRCT repeats and phosphoproteins: Tangled up in two. Trends Biochem. Sci. 29: 579-585.

Greenberg, R.A., Sobhian, B., Pathania, S., Cantor, S.B., Nakatani, Y., and Livingston, D.M. 2006. Multifactorial contributions to an acute DNA damage response by BRCA1/BARD1-containing complexes. Genes \& Dev. 20: 34-46.

Hashizume, R., Fukuda, M., Maeda, I., Nishikawa, H., Oyake, D., Yabuki, Y., Ogata, H., and Ohta, T. 2001. The RING heterodimer BRCA1-BARD1 is a ubiquitin ligase inactivated by a breast cancerderived mutation. J. Biol. Chem. 276: 14537-14540.

Kleiman, F.E., Wu-Baer, F., Fonseca, D., Kaneko, S., Baer, R., and Manley, J.L. 2005. BRCA1/BARD1 inhibition of mRNA 3' processing involves targeted degradation of RNA polymerase II. Genes \& Dev. 19: 12271237.

Lorick, K.L., Jensen, J.P., Fang, S., Ong, A.M., Hatakeyama, S., and Weissman, A.M. 1999. RING fingers mediate ubiquitin-conjugating enzyme (E2)-dependent ubiquitination. Proc. Natl. Acad. Sci. 96: 11364-11369.

Manke, I.A., Lowery, D.M., Nguyen, A., and Yaffe, M.B. 2003. BRCT repeats as phosphopeptide-binding modules involved in protein targeting. Science 302: 636-639.

Montes de Oca, R., Andreassen, P.R., Margossian, S.P., Gregory, R.C. Taniguchi, T., Wang, X., Houghtaling, S., Grompe, M., and D'Andrea, A.D. 2005. Regulated interaction of the Fanconi anemia protein, FANCD2, with chromatin. Blood 105: 1003-1009.

Morris, J.R. and Solomon, E. 2004. BRCA1:BARD1 induces the formation of conjugated ubiquitin structures, dependent on $\mathrm{K} 6$ of ubiquitin, in cells during DNA replication and repair. Hum. Mol. Genet. 13: 807817.

Nguyen, H.G., Chinnappan, D., Urano, T., and Ravid, K. 2005. Mechanism of Aurora-B degradation and its dependency on intact KEN and A-boxes: Identification of an aneuploidy-promoting property. Mol. Cell. Biol. 25: 4977-4992.

Rodriguez, M., Yu, X., Chen, J., and Songyang, Z. 2003. Phosphopeptide binding specificities of BRCAl COOH-terminal (BRCT) domains. $J$. Biol. Chem. 278: 52914-52918.

Ruffner, H., Joazeiro, C.A., Hemmati, D., Hunter, T., and Verma, I.M. 2001. Cancer-predisposing mutations within the RING domain of BRCA1: Loss of ubiquitin protein ligase activity and protection from radiation hypersensitivity. Proc. Natl. Acad. Sci. 98: 5134-5139.

Sato, K., Hayami, R., Wu, W., Nishikawa, T., Nishikawa, H., Okuda, Y., Ogata, H., Fukuda, M., and Ohta, T. 2004. Nucleophosmin/B23 is a candidate substrate for the BRCA1-BARD1 ubiquitin ligase. J. Biol. Chem. 279: 30919-30922.

Scully, R., Ganesan, S., Vlasakova, K., Chen, J., Socolovsky, M., and Livingston, D.M. 1999. Genetic analysis of BRCAl function in a defined tumor cell line. Mol. Cell 4: 1093-1099.

Starita, L.M., Machida, Y., Sankaran, S., Elias, J.E., Griffin, K., Schlegel, B.P., Gygi, S.P., and Parvin, J.D. 2004. BRCA1-dependent ubiquitination of $\gamma$-tubulin regulates centrosome number. Mol. Cell. Biol. 24: $8457-8466$.

Starita, L.M., Horwitz, A.A., Keogh, M.C., Ishioka, C., Parvin, J.D., and Chiba, N. 2005. BRCA1/BARD1 ubiquitinate phosphorylated RNA polymerase II. J. Biol. Chem. 280: 24498-24505.

Taniguchi, T., Garcia-Higuera, I., Andreassen, P.R., Gregory, R.C., Grompe, M., and D'Andrea, A.D. 2002. S-phase-specific interaction of the Fanconi anemia protein, FANCD2, with BRCA1 and RAD51. Blood 100: 2414-2420.

Tomlinson, G.E., Chen, T.T., Stastny, V.A., Virmani, A.K., Spillman, M.A., Tonk, V., Blum, J.L., Schneider, N.R., Wistuba, I.I., Shay, J.W., et al. 1998. Characterization of a breast cancer cell line derived from 
Yu et al.

a germ-line BRCA1 mutation carrier. Cancer Res. 58: 3237-3242.

Varma, A.K., Brown, R.S., Birrane, G., and Ladias, J.A. 2005. Structural basis for cell cycle checkpoint control by the BRCA1-CtIP complex. Biochemistry 44: 10941-10946.

Wang, X., Andreassen, P.R., and D'Andrea, A.D. 2004. Functional interaction of monoubiquitinated FANCD2 and BRCA2/FANCD1 in chromatin. Mol. Cell. Biol. 24: 5850-5862.

Ward, I.M. and Chen, J. 2001. Histone H2AX is phosphorylated in an ATR-dependent manner in response to replicational stress. J. Biol. Chem. 276: 47759-47762.

Wong, A.K., Ormonde, P.A., Pero, R., Chen, Y., Lian, L., Salada, G., Berry, S., Lawrence, Q., Dayananth, P., Ha, P., et al. 1998. Characterization of a carboxy-terminal BRCAl interacting protein. Oncogene 17: $2279-2285$.

Wu-Baer, F., Lagrazon, K., Yuan, W., and Baer, R. 2003. The BRCA1/ BARD1 heterodimer assembles polyubiquitin chains through an unconventional linkage involving lysine residue K6 of ubiquitin. J. Biol. Chem. 278: 34743-34746.

Xia, Y., Pao, G.M., Chen, H.W., Verma, I.M., and Hunter, T. 2003. Enhancement of BRCA1 E3 ubiquitin ligase activity through direct interaction with the BARD1 protein. J. Biol. Chem. 278: 5255-5263.

$\mathrm{Yu}, \mathrm{X}$. and Baer, R. 2000. Nuclear localization and cell cycle-specific expression of CtIP, a protein that associates with the BRCA1 tumor suppressor. J. Biol. Chem. 275: 18541-18549.

$\mathrm{Yu}, \mathrm{X}$. and Chen, J. 2004. DNA damage-induced cell cycle checkpoint control requires CtIP, a phosphorylation-dependent binding partner of BRCA1 C-terminal domains. Mol. Cell. Biol. 24: 9478-9486.

Yu, X., Wu, L.C., Bowcock, A.M., Aronheim, A., and Baer, R. 1998. The C-terminal (BRCT) domains of BRCAl interact in vivo with CtIP, a protein implicated in the CtBP pathway of transcriptional repression. J. Biol. Chem. 273: 25388-25392.

Yu, X., Chini, C.C., He, M., Mer, G., and Chen, J. 2003. The BRCT domain is a phospho-protein binding domain. Science 302: 639-642 


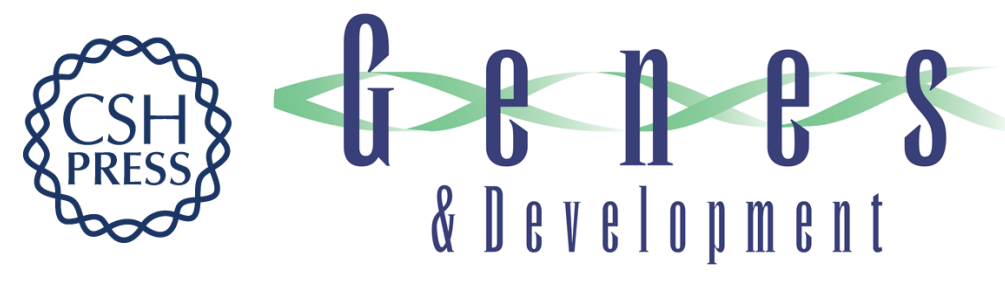

\section{BRCA1 ubiquitinates its phosphorylation-dependent binding partner CtIP}

Xiaochun Yu, Shuang Fu, Maoyi Lai, et al.

Genes Dev. 2006, 20:

Access the most recent version at doi:10.1101/gad.1431006

\section{Supplemental http://genesdev.cshlp.org/content/suppl/2006/06/09/20.13.1721.DC1 Material}

References This article cites 33 articles, 24 of which can be accessed free at: http://genesdev.cshlp.org/content/20/13/1721.full.html\#ref-list-1

\section{License}

Email Alerting

Receive free email alerts when new articles cite this article - sign up in the box at the top Service 